\title{
A systematic review of genetic skeletal disorders reported in Chinese biomedical journals between 1978 and 2012
}

\author{
Yazhou Cui ${ }^{1,2}$, Heng Zhao ${ }^{1,2}$, Zhenxing Liu ${ }^{1,2}$, Chao Liu ${ }^{1,2}$, Jing Luan ${ }^{1,2}$, Xiaoyan Zhou ${ }^{1,2}$ and Jinxiang Han ${ }^{1,2^{*}}$
}

\begin{abstract}
Little information is available on the prevalence, geographic distribution and mutation spectrum of genetic skeletal disorders (GSDs) in China. This study systematically reviewed GSDs as defined in "Nosology and Classification of genetic skeletal disorders (2010 version)" using Chinese biomedical literature published over the past 34 years from 1978 to 2012. In total, 16,099 GSDs have been reported. The most frequently reported disorders were Marfan syndrome, osteogenesis imperfecta, fibrous dysplasia, mucopolysaccharidosis, multiple cartilaginous exostoses, neurofibromatosis type 1 (NF1), osteopetrosis, achondroplasia, enchondromatosis (Ollier), and osteopoikilosis, accounting for $76.5 \%$ (12,312 cases) of the total cases. Five groups (group 8, 12, 14, 18, 21) defined by "Nosology and Classification of genetic skeletal disorders" have not been reported in the Chinese biomedical literature. Gene mutation testing was performed in only a minor portion of the 16,099 cases of GSDs (187 cases, 1.16\%). In total, 37 genes for 41 different GSDs were reported in Chinese biomedical literature, including 43 novel mutations. This review revealed a significant imbalance in rare disease identification in terms of geographic regions and hospital levels, suggesting the need to create a national multi-level network to meet the specific challenge of care for rare diseases in China.
\end{abstract}

Keywords: Rare diseases, Genetic skeletal diseases, China, Bibliographic study

\section{Introduction}

Genetic skeletal disorders (GSDs) arise from disturbances of the complex processes of skeletal development, growth, and homeostasis caused by gene mutations. These disorders represent a challenge in terms of diagnosis and treatment due to their rarity and variety $[1,2]$. The recently published "Nosology and Classification of Genetic skeletal disorders (2010 version)" listed 456 GSDs that were classified into 40 groups by clinical, radiographic, and molecular criteria; of these, 316 conditions were associated with mutations in 226 different genes [3]. The Nosology not only provides a guideline for the diagnosis of the patients and the recognition of the novel disorders for clinicians, but also is helpful for better understanding the mechanisms of

\footnotetext{
* Correspondence: samshjx@sina.com

${ }^{1}$ Shandong Academy of Medical Sciences, Shandong Medical Biotechnological Center, Jinan 250062, China

${ }^{2}$ Key Laboratory for Biotech Drugs of the Ministry of Health, Key Laboratory for Rare Disease Research of Shandong Province, Jinan 250062, China
}

genes, proteins and pathways involved in skeletal biology.

Until now, population-based studies to determine the prevalence of GSDs have been not been performed in China. Most GSDs have been reported in "case reports" in Chinese biomedical literature, but these sources are usually not available to international readers. Therefore, an introduction to the published literature on GSDs in China would enrich our knowledge on the prevalence and molecular characteristics of these rare diseases.

This study systematically reviewed GSDs reported in Chinese biomedical literature published over the past 34 years from January 1978 to January 2012. This study also analyzed the current state and specific challenges in diagnosing and treating rare diseases in China.

\section{Methods}

\section{Rare diseases covered}

This bibliographic study covered a total of 456 GSDs in 40 groups defined in "Nosology and Classification of

\section{Ciomed Central}


GSDs (2010 version)." This study has been performed with the approval of the ethics committee of Shandong Academy of Medical Science.

\section{Selection of database sources}

A literature search was conducted using China Biomedical Database (CBM) (http://sinomed.imicams.ac.cn) and covered sources from January 1978 to January 2012. The $\mathrm{CBM}$ is the largest Chinese biomedical bibliographic database [4], and includes a total of 6,840,907 articles from more than 1,600 biomedical journals published in Chinese prior to January 12, 2012.

\section{Search strategy}

The CBM database and public web search engines were first used to search for alternative Chinese terms for the English terms describing each disorder, and then all the terms for the disorder (both in English and Chinese) were used to search for publications in the CBM database. English terms for disorders were included since most Chinese biomedical articles contain an English abstract. The following search algorithm was used: "English disorder terms OR Chinese disorder terms [fulltext]". For diseases with different subtypes (for example, Osteogenesis Imperfecta, types $\mathrm{I}-\mathrm{V}$ ), only the main term ("Osteogenesis Imperfecta") was used in the search, and information on the type was gleaned from the text.

\section{Inclusion and exclusion criteria}

Clinical data and diagnostic information were gleaned from the abstract or full text of the articles searched for in the CBM database. Cases of GSDs with a confirmed diagnosis were included. Detailed clinical, imaging, and laboratory data needed to be described for case reports. Exact diagnostic criteria had to be included for research reports involving multiple cases or families. For each study included, informed consent to publication was obtained from the patient. Patient medical information was carefully compared for series of reports on the same disorders by the same authors or institutions, and redundant cases were excluded.

\section{Results}

According to our criteria 3,208 Chinese reports were qualified for inclusion. A total of 16,099 cases of GSDs in 35 groups of the "Nosology and Classification of Genetic Skeletal Disorders (2010 version)" was reported in the literature. The number of published cases is listed in Table 1. The 10 most frequently reported GSDs were Marfan syndrome, osteogenesis imperfecta, fibrous dysplasia, mucopolysaccharidosis, multiple cartilaginous exostoses, neurofibromatosis type 1 (NF1), osteopetrosis, achondroplasia, enchondromatosis (Ollier), and osteopoikilosis, accounting for $76.5 \%$ of cases (12,312 cases). Five groups (group 8 TRPV4 group, group 12 spondylometaphyseal dysplasias, group 14 severe spondylodysplastic dysplasias, group 18 bent bones dysplasias, and group 21 chondrodysplasia punctata) described in the Nosology have not been reported yet by Chinese biomedical literature.

The geographic distribution of cases is shown in Figure 1. GSDs have been reported in all of China's provinces and province-level municipalities. However, the number of cases varied geographically. More patients were reported in the East and South of China, which have a higher population density and better medical services than other areas. Beijing, Guangdong, Shandong, Shanghai and Jiangsu ranked among the top 5 provinces or provincelevel municipalities where disorders were reported.

As shown in Figure 2, the number of patients with GSDs reported each year in the CBM database increased gradually since 1978 and rapidly increased starting in 1994. Most GSD cases were reported by pediatricians, radiologists, and orthopedists. $49.0 \%$ of the cases were diagnosed at a university hospital,10.8\% were diagnosed at a provincial hospital, $32.7 \%$ were diagnosed at a municipal hospital, and the remainder (7.5\%) was diagnosed at hospitals on country level or even from smaller communities. (Figure 3).

Gene mutations were evaluated in 187 cases or families out of 16,099 total reported cases, accounting for only a minor portion (1.16\%). As shown in Table 2, a total of 37 genes for 41 different GSDs were reported, including 43 novel mutations that have not been reported before. The EXT1 and EXT2 genes (30 cases) for multiple cartilaginous exostoses, the FBN1 gene for Marfan syndrome (24 cases), and the FGFR3 gene for achondroplasia (22 cases) were most frequently reported in Chinese biomedical literature from the CBM database. Compared to the reported geographic distribution of GSDs (Figure 2), genetic testing was only performed at university hospitals in a few areas (Figure 4). Affiliated hospitals of Shanghai Jiaotong University, Chinese Academy Of Medical Science \& Peking Union Medical College, Zhongshan University, Central South University, and Peking University rank the top 5 University hospitals which performed most gene testing of GSDs.

\section{Discussion}

As the world's most densely populated nation, China has the world's largest number of rare disease groups [5]. In 1984, the concept of rare diseases was introduced in China. Until recently, however, the problem presented by rare diseases has received little attention [6]. Currently, there is no case registration system for most rare diseases, so there is very little documented information on the epidemiology of those diseases in China [7]. China still lacks an official definition and spectrum of rare diseases. 
Table 1 Number of published cases of genetic skeletal diseases in Chinese and Europe biomedical literature listed in alphabetical order of diseases*

\begin{tabular}{llrl}
\hline Diseases or group of diseases & $\begin{array}{l}\text { Number of Cases reported in } \\
\text { Chinese biomedical literature }\end{array}$ & $\begin{array}{c}\text { Number of published } \\
\text { cases in Europe }[8]\end{array}$ & $\begin{array}{c}\text { Estimated prevalence in } \\
\text { Europe }(/ 100,000)[8]\end{array}$
\end{tabular}

Achondroplasia

Acrofacial dysostosis, Nager type

90

alpha-Mannosidosis

Apert syndrome

Asphyxiating thoracic dysplasia

Brachydactyly

Caffey disease

Calcium pyrophosphate deposition disease (familial

Cartilage-hair hypoplasia (CHH; metaphyseal dysplasia,

McKusick type)

Cherubism

Chondrodysplasia punctata

Chondroectodermal dysplasia (Ellis-van Creveld)

Cleidocranial dysplasia

Congenital contractural arachnodactyly

Craniofrontonasal syndrome

Craniometaphyseal dysplasia

Craniostenosis (Craniosynostosis)

Crouzon syndrome

Currarino triad

de Lange syndrome

Diaphyseal dysplasia Camurati-Engelmann

Dysplasia epiphysealis hemimelica (Trevor)

Ectrodactyly-ectodermal dysplasia cleft-palate syndrome

Ehlers-Danlos syndrome

Enchondromatosis (Ollier)

Enchondromatosis with hemangiomata (Maffucci)

Endosteal hyperostosis, van Buchem type

Familial expansile osteolysis

Familial hip dysplasia (Beukes)

Familial osteochondritis dissecans

Fanconi anemia

Frontometaphyseal dysplasia

Frontonasal dysplasia

Fucosidosis

Fuhrmann syndrome

GM1 Gangliosidosis, several forms 
Table 1 Number of published cases of genetic skeletal diseases in Chinese and Europe biomedical literature listed in alphabetical order of diseases* (Continued)

Hallermann-Streiff syndrome

Hanhart syndrome(hypoglossia-hypodactylia)

Holt-Oram syndrome

Hypertrophic osteoarthropathy

Hypochondroplasia

Hypophosphatasia, perinatal lethal and infantile forms

Hypophosphatemic rickets

Idiopathic juvenile osteoporosis

Immuno-osseous dysplasia (Schimke)

Infantile systemic hyalinosis/Juvenile hyaline fibromatosis (ISH/JHF)

Klippel-Feil anomaly with laryngeal malformation

Kniest dysplasia

Langer type (Homozygous dyschondrosteosis)

Larsen syndrome

Lipomembraneous osteodystrophy with

leukoencephalopathy (presenile dementia with

bone cysts; Nasu-Hakola)

Mandibulo-facial dysostosis(Treacher-Collins,

Franceschetti-Klein)

Marfan syndrome

Marshall syndrome

Meckel syndrome

Melorheostosis

Melorheostosis with osteopoikilosis

Mesomelic dysplasia

Metaphyseal dysplasia, Jansen type

Metaphyseal dysplasia, Schmid type (MCS)

Mucolipidosis II (I-cell disease),alpha/beta type

Mucopolysaccharidosis

Multicentric carpal-tarsal osteolysis with and without nephropathy

Multiple cartilaginous exostoses

Multiple epiphyseal dysplasia

Multiple sulfatase deficiency

Multiple synostoses syndrome

Nail-patella syndrome

Neonatal hyperparathyroidism, severe form

Neurofibromatosis type 1 (NF1)

Oculodentoosseous dysplasia

Omodysplasia

Oral-facial-digital syndrome

Osteoectasia with hyperphosphatasia (juvenile

Paget disease)

Osteogenesis imperfecta

32

$<100$

$<50$
N.A.

100

N.A.

N.A. 
Table 1 Number of published cases of genetic skeletal diseases in Chinese and Europe biomedical literature listed in alphabetical order of diseases* (Continued)

\begin{tabular}{|c|c|c|c|}
\hline Osteopetrosis & 810 & & 1.75 \\
\hline Osteopoikilosis & 338 & 300 & \\
\hline $\begin{array}{l}\text { Pachydermoperiostosis (hypertrophic osteoarthropathy, } \\
\text { primary, autosomal dominant) }\end{array}$ & 25 & 204 & \\
\hline Pallister-Hall syndrome & 1 & 100 & \\
\hline Parietal foramina & 11 & & 5 \\
\hline Pfeiffer syndrome & 4 & & 1 \\
\hline Poland anomaly & 27 & 3 & \\
\hline Preaxial polydactyly & 16 & & 25 \\
\hline Progeria, Hutchinson-Gilford type & 20 & & 0.005 \\
\hline Progressive osseous heteroplasia & 20 & N.A. & \\
\hline $\begin{array}{l}\text { Progressive pseudorheumatoid dysplasia (PPRD; SED with } \\
\text { progressive arthropathy) }\end{array}$ & 6 & N.A. & \\
\hline Proteus syndrome & 22 & 200 & \\
\hline Proximal symphalangism & 15 & N.A. & \\
\hline Pseudoachondroplasia (PSACH) & 51 & & 1.6 \\
\hline Pyknodysostosis & 14 & & 0.13 \\
\hline Pyle disease & 2 & $<30$ & \\
\hline Radio-ulnar synostosis & 55 & $<20$ & \\
\hline Saethre-Chotzen syndrome & 1 & & 3 \\
\hline Schwartz-Jampel syndrome (myotonic chondrodystrophy) & 4 & 100 & \\
\hline SED tarda, X-linked (SED-XL) & 156 & & 0.55 \\
\hline SED, Wolcott-Rallison type & 1 & $<60$ & \\
\hline Short rib-polydactyly syndrome & 57 & N.A. & \\
\hline Shprintzen-Goldberg syndrome & 1 & $<50$ & \\
\hline Sotos syndrome & 38 & & 7 \\
\hline Split hand-foot malformation & 25 & & 1.1 \\
\hline Spondylocostal dysostosis & 1 & 4 & \\
\hline Spondyloepiphyseal dysplasia congenita (SEDC) & 50 & & 0.34 \\
\hline Spondylometaphyseal dysplasia & 6 & & 1 \\
\hline Spondylometaphyseal dysplasia, Kozlowski type & 2 & & 0.1 \\
\hline $\begin{array}{l}\text { Sterile multifocal osteomyelitis,periostitis, and pustulosis } \\
\text { (CINCA/NOMID-like) }\end{array}$ & 1 & N.A. & \\
\hline Stickler syndrome & 7 & & 13.5 \\
\hline Syndactyly type 5 (HOXD13) & 7 & N.A. & \\
\hline Thanatophoric dysplasia & 28 & & 3.5 \\
\hline Thrombocytopenia-absent radius & 1 & N.A. & \\
\hline Tibial hemimelia & 2 & & 0.1 \\
\hline Trichorhinophalangeal dysplasia & 15 & $>100$ & \\
\hline
\end{tabular}

*: Different types belong to one diseases have been combined as one item when the typing information was not provided in literature.

A bibliographic study will help to estimate the prevalence of rare diseases [8]. Most rare diseases have been reported in Chinese biomedical publications. Presently, only 86 Chinese biomedical journals have abstracts in English included in Pubmed [9]. Therefore, most reports on rare diseases in China are unavailable to international readers. To our knowledge, the current study is the first systematic review of the Chinese biomedical literature on rare disease groups.

GSDs are representative for many other groups of rare diseases. The current systematic review found that the number and type of GSDs reported in Chinese biomedical 

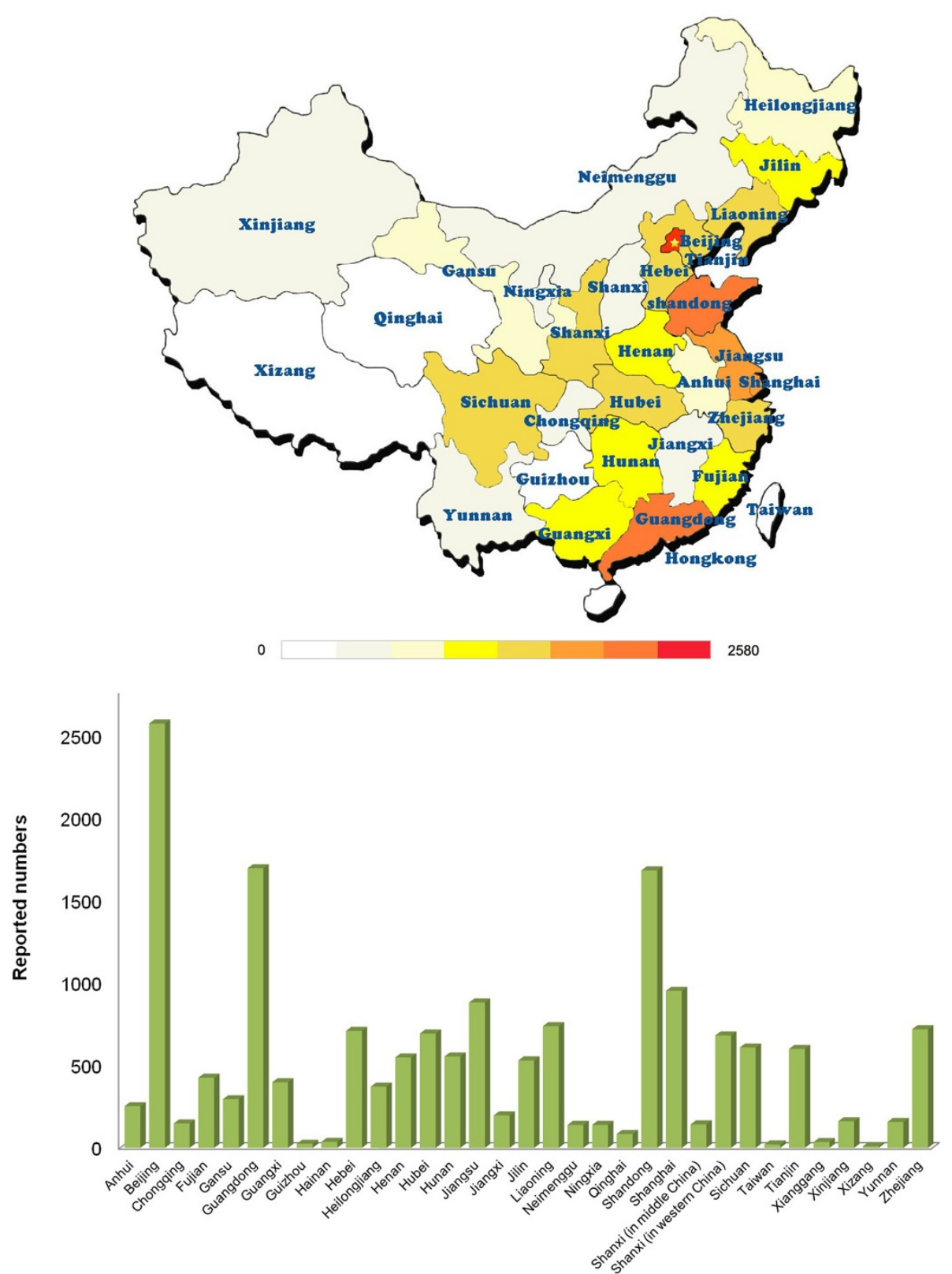

Figure 1 Geographic distribution of reported cases of genetic skeletal disorders (GSDs) in Chinese biomedical literature. The number of cases reported varied geographically, and focused in the East and South of China. Beijing, Guangdong, Shandong, Shanghai and Jiangsu ranked among the top 5 provinces or province-level municipalities where GSDs were reported.

literature increased gradually over the past 30 years. In the last 5 years in particular, there were 1,057 cases reported annually, which is due to the rapid improvement of general healthcare and increasing attention to the medical problems caused by rare diseases in China. Although most genetic skeletal disease groups have been reported in Chinese biomedical literature, but only a small portion of patients were exactly molecularly characterized. For example, 1,314 cases of osteogenesis imperfecta were reported in the CBM database but in only $5 \%$ the exact type has been determined. This situation might be mainly due to the fact that most of these patients were diagnosed based on clinical and radiographic criteria and because gene mutation testing has been unavailable at most hospitals until now. In only $1 \%$ of all cases with GSDs a causative gene mutation was identified. Among these reported mutations, there is a relatively high frequency of novel mutations. These novel variations may also lead to a better understanding of the mutation spectrum and impact of genes associated with GSDs. For example, 5 cases of 


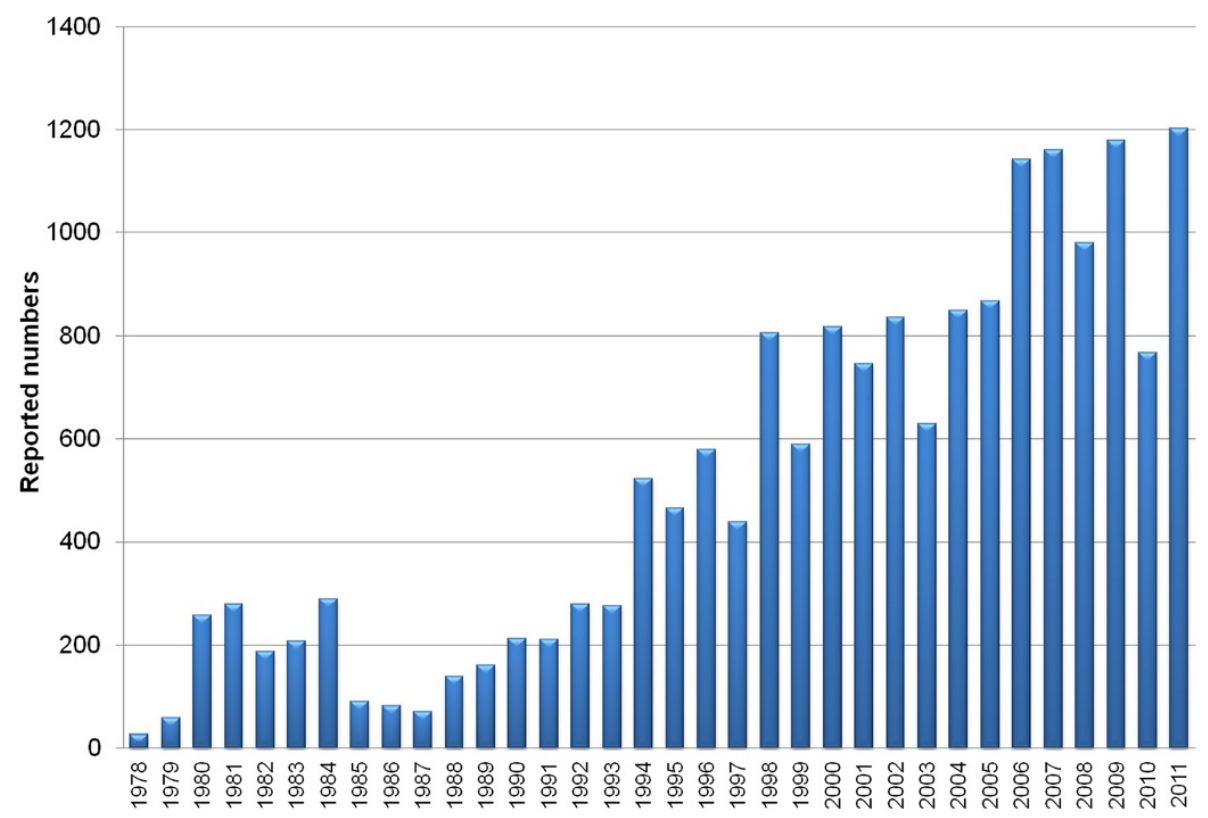

Figure 2 Case number of genetic skeletal disorders reported in Chinese biomedical literatures from 1978 to 2011. The number of GSDs reported each year in the CBM database increased gradually since 1978 and rapidly increased starting in 1994. 1,057 cases were reported annually in recent 5 years.

novel mutations in COL1A1 have been reported in Chinese biomedical literature. Among them, 4 cases belong to glycine single base substitution mutations in the triplehelical region (p.G632x, p.G1157D) and splicing sites (IVS27 + $1 \mathrm{G}>\mathrm{A}$, IVS8-2A $>\mathrm{G}$ ), which are the most and second common mutation types in COL1A1 gene. Mutations in the $\mathrm{C}$-propeptide coding region have been identified less frequent than other forms of mutation. D1441 is one of a few residues absolutely conserved in this region,

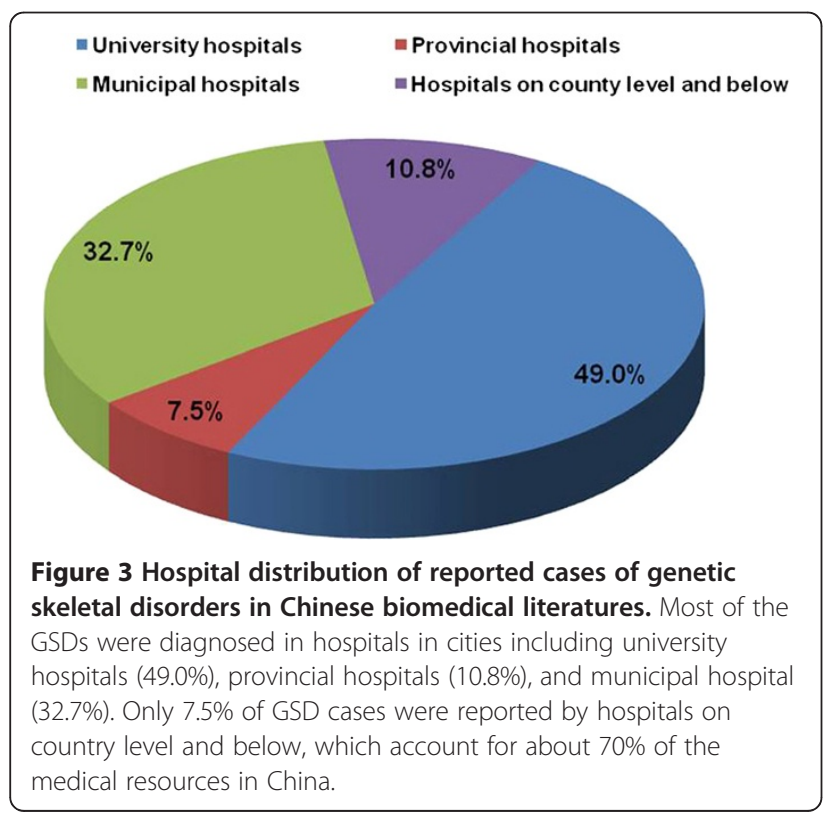

a previous study reported a defect in this site (D1441Y) resulting in a lethal variant of osteogenesis imperfecta with features of dense bone diseases [10], however, a novel mutation in this site described in a Chinese family (D1441H) led to only mild osteogenesis imperfecta (type 1), which suggesting mutations in this region show great heterogeneity in clinical outcome.

This systematic review of genetic skeletal diseases also revealed that reporting of rare diseases varies significantly in different regions and medical resources available in China. Cases of rare diseases were more frequently reported in large municipalities such as Beijing and Shanghai instead of areas with a larger population but a relatively lower level of development such as Sichuan and Henan Provinces. Country level hospitals and below are mainly responsible for treating rural residents and represent more than $70 \%$ of the medical resources in China [11]. In this review, we found that only $7.5 \%$ of the cases of rare diseases were diagnosed by these hospitals, which is significantly lower than that diagnosed by urban hospitals. In actuality, there are also significant disparities in health care between university hospitals and provincial and municipal hospitals. Currently, gene mutation testing for genetic rare diseases in China is done only by university hospitals in several key municipalities.

We further compared the number of GSD cases reported in Chinese biomedical literature with those of published cases or estimated prevalence of these diseases in Europe from bibliographic data issued by Orphanet 
Table 2 Gene mutation of genetic skeletal disorders published in Chinese biomedical literature from 1978 to $2012 *$

\begin{tabular}{|c|c|c|c|c|c|c|c|}
\hline Gene & Name of disorder & MIM No. & $\begin{array}{l}\text { No. of } \\
\text { case } \\
\text { reported* }\end{array}$ & Mutation & Location & Type & Novel \\
\hline \multirow[t]{2}{*}{ ACVR1 } & Fibrodysplasia & 135100 & 2 & c.617 G > A (p.R206H) & exon 4 & missense & \\
\hline & & & 1 & c.1067 G > A (p.G356D) & exon 7 & missense & \\
\hline ALPL & $\begin{array}{l}\text { Hypophosphatasia, } \\
\text { infantile forms }\end{array}$ & 241500 & 1 & $\begin{array}{l}\text { c.18delA and c.G407C } \\
\text { (p.V7Yfs18X and p.R136P) }\end{array}$ & exon 2 and 5 & $\begin{array}{l}\text { nonsense } \\
\text { and missense }\end{array}$ & yes \\
\hline \multirow[t]{3}{*}{ ALPL } & $\begin{array}{l}\text { Hypophosphatasia, } \\
\text { adult form }\end{array}$ & 146300 & 1 & c.1366 G > A (p.G456R) & exon 12 & missense & \\
\hline & & & 1 & c.1581C > G (p.P446G) & exon 12 & missense & yes \\
\hline & & & 1 & c.583 G>A (p.R136H) & exon 5 & missense & \\
\hline \multirow[t]{6}{*}{ CLCN5 } & Dent's disease & 300554 & 1 & p.L594fsX595 & exon 10 & nonsense & yes \\
\hline & & & 1 & p.R637X & exon 10 & nonsense & \\
\hline & & & 1 & p.R467X & exon 9 & nonsense & \\
\hline & & & 1 & p.IVS4-2A > G & exon 4 & splicing & yes \\
\hline & & & 1 & c.1022C > T (p.S244L) & exon 7 & missense & \\
\hline & & & 1 & c.1805 T> G (p.V505G) & exon 9 & missense & yes \\
\hline CLCN7 & $\begin{array}{l}\text { Osteopetrosis, } \\
\text { late-onset form type } 2 \\
\text { (OPTA2) }\end{array}$ & 166600 & 1 & c.C856T (p.R286W) & exon 10 & missense & \\
\hline \multirow[t]{2}{*}{ COL1A2 } & $\begin{array}{l}\text { Osteogenesis } \\
\text { imperfecta }\end{array}$ & & 1 & c.A3350G (p.Y1117C) & exon 49 & missense & \\
\hline & & & 1 & c.G3305C (p.G1 102A) & exon 49 & missense & \\
\hline \multirow[t]{7}{*}{ COL1A1 } & $\begin{array}{l}\text { Osteogenesis } \\
\text { imperfecta }\end{array}$ & 166220 & 1 & c.1678 G > A (p.G560S) & exon 25 & missense & \\
\hline & & 166200 & 1 & p.Gly632x & exon 28 & nonsense & yes \\
\hline & & 166200 & 1 & p.D1441H & exon 52 & missense & yes \\
\hline & & 166200 & 1 & c. $1875+1 \mathrm{G}>\mathrm{A}($ IVS $27+1 \mathrm{G}>\mathrm{A})$ & intron 27 & splicing & yes \\
\hline & & 166200 & 1 & IVS8-2A $>G$ & intron 8 & splicing & yes \\
\hline & & & 1 & c.2461 G > A (p.G821S) & exon 36 & missense & \\
\hline & & & 1 & c.3470 G > A (p.G1157D) & & missense & yes \\
\hline \multirow[t]{2}{*}{ COL2A1 } & Spondyloepiphyseal & 183900 & 1 & c. 1510 G > A (p.G504S) & & missense & \\
\hline & & & 1 & c.2401 G > A (p.G801S) & & missense & \\
\hline EFNB1 & $\begin{array}{l}\text { Craniofrontonasal } \\
\text { Syndrome }\end{array}$ & 304110 & 1 & c.161C > G (p.P54R) & exon 2 & missense & yes \\
\hline COMP & Pseudoachondroplasia & 177170 & 1 & c.815 T>C (p.L272P) & exon 8 & missense & \\
\hline EIF2AK3 & $\begin{array}{l}\text { SED, Wolcott-Rallison } \\
\text { type }\end{array}$ & 226980 & 1 & $\begin{array}{l}\text { c.1408-1409insT and c.1596 T > A } \\
\text { (p.S470FfsX7 and p.C532X) }\end{array}$ & exon 8 and 9 & nonsense & \\
\hline \multirow[t]{8}{*}{ EXT1 } & $\begin{array}{l}\text { Multiple cartilaginous } \\
\text { exostoses } 1\end{array}$ & 133700 & 1 & c.1564-7delC & exon 7 & frameshift & yes \\
\hline & & & 1 & $18+2 T>G$ & intron 8 & splicing & yes \\
\hline & & & 1 & c.651_664delinsTTT (p.K218fsX220) & exon 1 & nonsense & \\
\hline & & & 1 & c.680delG (p.R227fs) & exon 1 & frameshift & \\
\hline & & & 1 & c.1182delG (p.Arg394SerfsX9) & exon 4 & nonsense & yes \\
\hline & & & 1 & c.1108 G > T (p.E370X) & exon 3 & nonsense & yes \\
\hline & & & 1 & c.335delA (p.Asn112ThrfsX24) & exon 1 & nonsense & yes \\
\hline & & & 1 & c.361C > T (p.Q121X) & exon 1 & nonsense & yes \\
\hline
\end{tabular}


Table 2 Gene mutation of genetic skeletal disorders published in Chinese biomedical literature from 1978 to $2012^{*}$ (Continued)

\begin{tabular}{|c|c|c|c|c|c|c|c|}
\hline & & & 1 & c.1879_1881delCAC (p.His627del) & exon 9 & In frame deletion & yes \\
\hline & & & 1 & c.651_664delinsTTT (p.K218fsX220) & exon 1 & frameshift & \\
\hline & & & 1 & $1633-26(C>A)$ & intron 7 & splicing & \\
\hline & & & 1 & c.2120delT & exon 6 & frameshift & \\
\hline & & & 1 & c.811 T> C (p.Y271H) & exon 1 & missense & yes \\
\hline \multirow[t]{15}{*}{ EXT2 } & $\begin{array}{l}\text { Multiple cartilaginous } \\
\text { exostoses } 2\end{array}$ & 133701 & 1 & c.668 G > C (p.Arg223Pro) & exon 2 & missense & \\
\hline & & & 1 & c.950delT (p.Phe317SerfsX15) & exon 6 & nonsense & yes \\
\hline & & & 3 & c.1016 G > A (p.Cys339Tyr) & exon 6 & missense & \\
\hline & & & 1 & c.398 T> G (p.L133R) & exon 2 & missense & \\
\hline & & & 1 & c.751C > T (p.Q251X) & exon 5 & missense & \\
\hline & & & 1 & c.544C > T (p.R182X) & exon 3 & missense & \\
\hline & & & 1 & c.536 G > A (p.Arg179Lys) & exon 2 & missense & \\
\hline & & & 1 & c.1006C > T (p.G1n336X) & exon 6 & nonsense & yes \\
\hline & & & 1 & IVS2 $+1 \mathrm{G}>\mathrm{A}$ & intron 2 & splicing & \\
\hline & & & 1 & IVS7 + $1 \mathrm{G}>\mathrm{T}$ & intron 7 & splicing & \\
\hline & & & 1 & c.789-796delTGTT & exon 5 & frameshift & yes \\
\hline & & & 1 & c.637 G > A & exon 4 & nonsense & \\
\hline & & & 1 & c.313A > T (p.Lys105X) & exon 2 & nonsense & \\
\hline & & & 1 & 319insGT & exon 2 & frameshift & \\
\hline & & & 1 & $536+1 \mathrm{G}>\mathrm{A}(\mathrm{IVS} 2+1 \mathrm{G}>\mathrm{A})$ & intron 2 & splicing & \\
\hline \multirow[t]{17}{*}{ FBN1 } & Marfan syndrome & 154700 & 1 & c.3463 G > A (p.Asp1155Asn) & exon 27 & missense & yes \\
\hline & & & 1 & c.5015 G > C (p.C1672S) & exon 40 & missense & \\
\hline & & & 3 & c.5309 G > A (p.C1770Y) & exon 43 & missense & \\
\hline & & & 2 & c.7241 G > A (p.R2414Q) & exon 58 & missense & \\
\hline & & & 2 & c.7769 G > A (p.C2590Y) & exon 62 & missense & \\
\hline & & & 2 & c.2261A > G (p.Y754C) & exon 18 & missense & \\
\hline & & & 1 & $\begin{array}{l}\text { c.[6862_6871delGGCTGTGTAG; } \\
6871+1 \text { 6871+ 11delGTAAGAGGATC] } \\
\text { (p.Gly2288MetfsX109) }\end{array}$ & exon 55 & nonsense & yes \\
\hline & & & 1 & c.2462 G > A (p.Cys821Tyr) & exon 20 & missense & yes \\
\hline & & & 1 & c.5015 G > C (p.C1672S) & exon 40 & missense & \\
\hline & & & 1 & c.3295 G > T (p.E1099X) & exon 26 & nonsense & \\
\hline & & & 2 & c.4307insTCGT (p.G1441X) & exon 34 & nonsense & yes \\
\hline & & & 1 & $c .4621 C>T(p . R 1541 X)$ & exon 37 & nonsense & \\
\hline & & & 1 & $c .8080 C>T(p . A 2694 X)$ & exon 64 & nonsense & \\
\hline & & & 2 & IVS29+4A > T & intron 29 & splicing & \\
\hline & & & 1 & IVS50 + $1 \mathrm{G}>\mathrm{A}$ & intron 50 & splicing & \\
\hline & & & 1 & c.3069 G > T (p.Lys1023Asn) & exon 24 & missense & yes \\
\hline & & & 1 & c.3243-3256delGCCTCTGCACCCA & exon 25 & frameshift & \\
\hline FGFR1 & Pfeiffer syndrome & 101600 & 1 & c.755C > G (p.Pro252Arg) & exon 5 & missense & \\
\hline \multirow[t]{5}{*}{ FGFR2 } & Pfeiffer syndrome & 101600 & 1 & IVS8 $A>G$ & exon 8 & splicing & \\
\hline & & & 1 & p.Asp321Ala & exon 9 & missense & \\
\hline & Apert syndrome & 101200 & 1 & c. $.934 C>$ G (p.S252W) & exon 7 & missense & \\
\hline & Crouzon syndrome & 123500 & 1 & c.833 G > T (p.C278F) & exon 8 & missense & \\
\hline & & & 1 & p.Tyr340His & exon 9 & missense & \\
\hline
\end{tabular}


Table 2 Gene mutation of genetic skeletal disorders published in Chinese biomedical literature from 1978 to $2012^{*}$ (Continued)

\begin{tabular}{|c|c|c|c|c|c|c|c|}
\hline & & & 1 & p.Cys342Trp & exon 9 & missense & yes \\
\hline & & & 1 & p.Cys342Tyr & exon 9 & missense & \\
\hline & & & 1 & p.Ala344Ala & exon 9 & missense & \\
\hline & & & 1 & p.Gly338Arg & exon 9 & missense & \\
\hline & & & 1 & p.Ala344Gly & exon 9 & missense & \\
\hline & & & 1 & p.Gln289Pro & exon 7 & missense & \\
\hline FGFR3 & Achondroplasia & 100800 & 21 & c.1138 G > A (p.G380R) & exon 10 & missense & \\
\hline & & & 1 & p.Ser217Cys & exon 5 & missense & \\
\hline FGFR3 & $\begin{array}{l}\text { Thanatophoric } \\
\text { dysplasia }\end{array}$ & 187600 & 1 & c.742C > T (p.R248C) & exon 7 & missense & \\
\hline GALNS & $\begin{array}{l}\text { Mucopolysaccharidosis } \\
\text { type 4A }\end{array}$ & 253000 & 1 & c. $1567 \mathrm{~T}>\mathrm{G}$ and c.374C > T & exon 14 and 4 & $\begin{array}{l}\text { nonsense } \\
\text { and missense }\end{array}$ & yes \\
\hline GDF5 & $\begin{array}{l}\text { Multiple synostoses } \\
\text { syndrome type } 2\end{array}$ & 186500 & 2 & c.1471 G > A (p.E491K) & exon 2 & missense & \\
\hline GNAS1 & $\begin{array}{l}\text { Albright hereditary } \\
\text { osteodystrophy }\end{array}$ & 103580 & 1 & 1-bp (C) deletion at codon 291 & exon 11 & frameshift & \\
\hline HLXB9 & Currarino triad & 176450 & 2 & c.552C > G (p.Tyr184X) & & nonsense & \\
\hline TP63 & $\begin{array}{l}\text { Split hand-foot } \\
\text { malformation, isolated } \\
\text { form, type } 4 \text { (SHFM4) }\end{array}$ & 605289 & 1 & $c .956 \mathrm{G}>\mathrm{A}(\mathrm{p} . \mathrm{R} 280 \mathrm{H})$ & exon 7 & missense & \\
\hline HOXD13 & Synpolydactyly & 186000 & 1 & c.32 G > C (p.G11A) & exon 1 & missense & \\
\hline & & & 1 & c.64 G > T (p.A22S) & exon 1 & missense & yes \\
\hline & & & 3 & 9-residue polyalanine expansion & exon 1 & & \\
\hline & yes & & & & & & \\
\hline & & & 1 & 8-residue polyalanine expansion & exon 1 & & \\
\hline & & & 1 & 7-residue polyalanine expansion & exon 1 & & \\
\hline IDS & $\begin{array}{l}\text { Mucopolysaccharidosis } \\
\text { type } 2\end{array}$ & 309900 & 1 & c.892C > T (p.Q298X) & exon 7 & nonsense & \\
\hline & & & 1 & c.1468delA & exon 9 & frameshift & \\
\hline & & & 1 & c.263 G > A (p.Arg88His) & exon 3 & missense & \\
\hline & & & 1 & 1103_1123del19 & exon 8 & frameshift & \\
\hline $\mathrm{IHH}$ & Brachydactyly type A1 & 112500 & 1 & c.G298A (p.D100N) & exon 1 & missense & \\
\hline NF1 & Neurofibromatosis type 1 & 162200 & 1 & c. $1009 \mathrm{G}>\mathrm{T}$ & exon 7 & nonsense & yes \\
\hline & & & 1 & c.3443-3444delCA & exon 20 & frameshift & yes \\
\hline & & & 1 & c.4339C > T (p.G1336X) & & nonsense & \\
\hline & & & 1 & c.5839C > T (p.R1947X) & exon 31 & nonsense & \\
\hline & & & 1 & p.Leu1141Arg & exon 20 & missense & \\
\hline TP63 & $\begin{array}{l}\text { Ankyloblepharon- } \\
\text { ectodermal dysplasia- } \\
\text { cleft lip/palate }\end{array}$ & 106260 & 1 & $c .838 \mathrm{C}>\mathrm{T}(\mathrm{p} . \mathrm{R} 280 \mathrm{C})$ & exon 7 & missense & \\
\hline & $\begin{array}{l}\text { Limb-mammary } \\
\text { syndrome (including } \\
\text { ADULT syndrome) }\end{array}$ & 603273 & 1 & c.893 G > A (p.R298Q) & exon 8 & missense & \\
\hline PHEX & $\begin{array}{l}\text { Hypophosphatemic } \\
\text { rickets, X-linked } \\
\text { dominant }\end{array}$ & 307800 & 1 & IVS20-1 G > T & intron 20 & splicing & \\
\hline & & & 1 & c.1861C > T (p.Gln621X) & exon 18 & missense & yes \\
\hline PTPN11 & $\begin{array}{l}\text { Baller-Gerold } \\
\text { syndrome }\end{array}$ & 218600 & 1 & IVS11-1 G > A and c.3401A > T & $\begin{array}{l}\text { intro } 11 \\
\text { and exon } 10\end{array}$ & $\begin{array}{l}\text { splicing } \\
\text { and nonsense }\end{array}$ & \\
\hline
\end{tabular}


Table 2 Gene mutation of genetic skeletal disorders published in Chinese biomedical literature from 1978 to $2012^{*}$ (Continued)

\begin{tabular}{|c|c|c|c|c|c|c|c|}
\hline \multirow[t]{2}{*}{ ROR2 } & \multirow[t]{2}{*}{ Brachydactyly type B } & \multirow[t]{2}{*}{113000} & \multirow{2}{*}{$\begin{array}{l}1 \\
1\end{array}$} & \multirow{2}{*}{$\begin{array}{l}\text { c.2265C > A (p.Y755X) } \\
\text { c.1398-1399insA }\end{array}$} & \multirow{2}{*}{$\begin{array}{l}\text { exon } 9 \\
\text { exon } 9\end{array}$} & \multirow{2}{*}{$\begin{array}{l}\text { nonsense } \\
\text { nonsense }\end{array}$} & \\
\hline & & & & & & & \\
\hline \multirow[t]{7}{*}{ RUNX2 } & Cleidocranial dysplasia & 119600 & 1 & c.346 T>A (p.W116R) & exon 1 & missense & \\
\hline & & & 1 & c.610A > T (p.K204X) & exon 3 & nonsense & \\
\hline & & & 1 & c.346 T>A (p.W116R) & exon 1 & missense & \\
\hline & & & 1 & c.475 G > C (p.G159R) & exon 2 & missense & yes \\
\hline & & & 1 & $c .673 C>T(p . R 225 W)$ & exon 3 & missense & \\
\hline & & & 1 & c.1171C > G (p.R391X) & exon 7 & nonsense & \\
\hline & & & 1 & c.674 G > A (p.R225Q) & exon 3 & missense & \\
\hline SALL1 & $\begin{array}{l}\text { Townes-Brocks } \\
\text { syndrome (Renal-Ear- } \\
\text { Anal-Radial syndrome) }\end{array}$ & 107480 & 4 & c. $1792 \mathrm{G}>\mathrm{C}$ & exon 2 & missense & \\
\hline \multirow[t]{9}{*}{ SEDL } & $\begin{array}{l}\text { SED tarda, X-linked } \\
\text { (SED-XL) }\end{array}$ & 313400 & 1 & c.218C > T (p.S73L) & exon 4 & missense & \\
\hline & & & 1 & c.370-371insA (p.S124fsX127) & exon 6 & nonsense & yes \\
\hline & & & 1 & c.218C > T (p.S73L) & exon 4 & missense & \\
\hline & & & 1 & c.239A > G (p.H80R) & exon 4 & missense & \\
\hline & & & 1 & c.G209A & exon 4 & nonsense & \\
\hline & & & 1 & c.262-266delGACAT & exon 5 & frameshift & \\
\hline & & & 1 & D109-S123del (p.S124fsX126) & intron 5-exon 6 & nonsense & \\
\hline & & & 1 & IVS5-2-1 delAG322-332delTTITCAATGAA & intron 5-exon 6 & splicing & yes \\
\hline & & & 1 & IVS2-2A >C & intron 2 & & \\
\hline \multirow[t]{2}{*}{ SH3BP2 } & Cherubism & 118400 & 5 & c.1505 G > C (p.Arg415Pro) & exon 9 & missense & \\
\hline & & & 2 & c.G1520A (p.Gly420Glu) & exon 9 & missense & \\
\hline \multirow[t]{3}{*}{ SHOX } & Dyschondrosteosis & 127300 & 1 & c. $115 \mathrm{~T}>\mathrm{G}$ & exon 2 & & \\
\hline & & & 1 & c.1171-1172insA & exon 3 & frameshift & \\
\hline & & & 1 & c.996A > T (p.E102V) & exon 3 & missense & \\
\hline SOX9 & $\begin{array}{l}\text { Campomelic dysplasia } \\
\text { (CD) }\end{array}$ & 114290 & 1 & p.R178L & exon 2 & missense & yes \\
\hline \multirow[t]{3}{*}{ TBX5 } & Holt-Oram syndrome & 142900 & 1 & c.416delC & exon 4 & frameshift & \\
\hline & & & 1 & C. $145 C>A$ & exon 2 & missense & \\
\hline & & & 1 & C.161 T>C & exon 2 & missense & \\
\hline TGFbeta1 & $\begin{array}{l}\text { Diaphyseal dysplasia } \\
\text { Camurati-Engelmann }\end{array}$ & 131300 & 1 & p.R218H & exon 4 & missense & \\
\hline \multirow[t]{4}{*}{ WISP3 } & $\begin{array}{l}\text { Progressive } \\
\text { pseudorheumatoid } \\
\text { dysplasia }\end{array}$ & 208230 & 1 & $\begin{array}{l}\text { c.624-625insA and } \\
\text { c.729-735delGAGAAAA }\end{array}$ & $\begin{array}{l}\text { exon } 4 \\
\text { and exon } 4\end{array}$ & $\begin{array}{l}\text { frameshift } \\
\text { and frameshift }\end{array}$ & yes \\
\hline & & & 1 & c.624-625insA and c.866-867insA & $\begin{array}{l}\text { exon } 4 \\
\text { and exon } 5\end{array}$ & $\begin{array}{l}\text { frameshift } \\
\text { and frameshift }\end{array}$ & yes \\
\hline & & & 1 & c.866_867insA and c.866-867insA & $\begin{array}{l}\text { exon } 5 \\
\text { and exon } 5\end{array}$ & $\begin{array}{l}\text { frameshift } \\
\text { and frameshift }\end{array}$ & yes \\
\hline & & & 1 & c. $589+2 \mathrm{~T}>\mathrm{C}$ and c.624dupA & $\begin{array}{l}\text { intro } 3 \\
\text { and exon } 4\end{array}$ & $\begin{array}{l}\text { splicing } \\
\text { and nonsense }\end{array}$ & yes \\
\hline
\end{tabular}

*: "Pedigree mutation", that is an identical mutation has been reported in more than one affected siblings in a family, was counted as one case. Mutation information was extracted from the full text, as its original description, all the novel mutations were claimed in the papers by the authors, and then were confirmed by searching the previous literature and the Human Gene Mutation Database.

[8] (Table 1). Generally, the number of GSDs reported in Chinese biomedical literatures is lower than in Europe, but, with some exceptions, the proportions between the different entities are similar. One of these exceptions are the multiple epiphyseal dysplasias, whose frequency in Europe is 5/100,000, while only a total of 122 cases were 


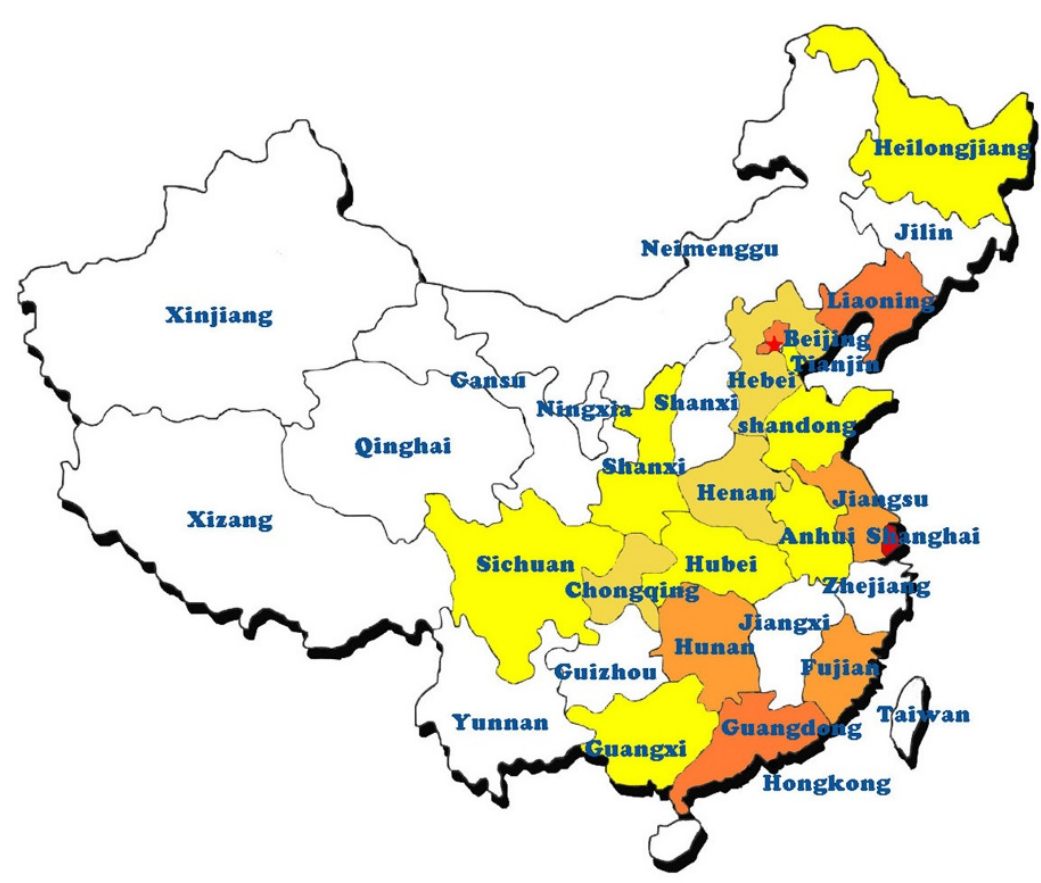

0

31

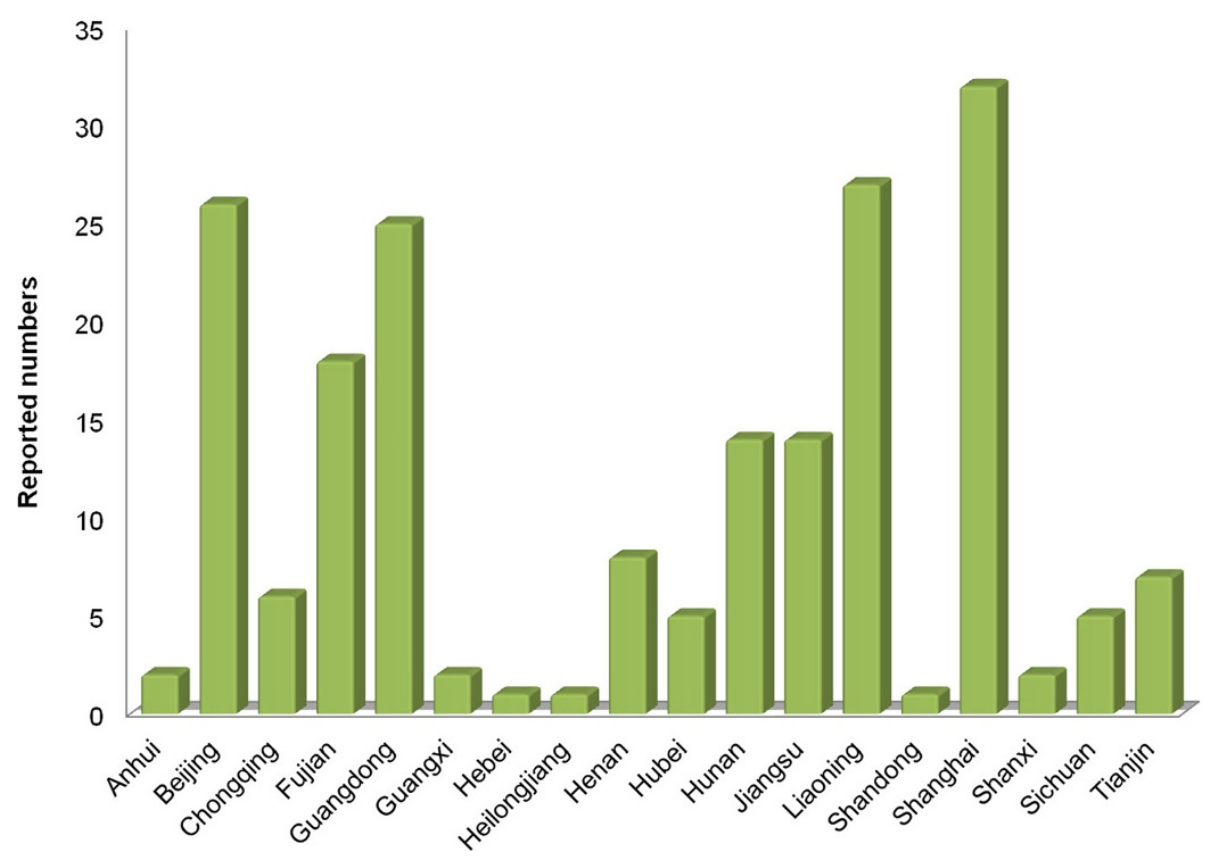

Figure 4 Geographic distribution of reported genetic skeletal disorders with gene mutation testing in Chinese biomedical literature. Genetic testing for GSDs was only performed at university hospitals in a few areas, far less than the regions where GSDs were reported.

reported in Chinese biomedical literature in the past 34 years. Although publication bias and genetic differences between Caucasian and Asian people may exist, we think this discrepancy is mainly due to the fact that China is still lagging behind Europe in terms of the medical resources for these rare diseases, especially in the widespread underdeveloped regions and hospitals on basic levels, therefore, many patients with genetic skeletal disorders could not acquire proper and timely diagnosis in China. 
Creating a network for rare diseases is an important medical policy that should significantly reduce misdiagnosis and improve the level of treatment. A network for collaboration with national medical resources has been set up in countries and regions such as Europe, North America, and Japan $[12,13]$. A number of centers offering counseling on rare diseases have been established in major Chinese cities and several provinces, but a national network has yet to be created. Given the fact that there is a huge gap in terms of medical services in different areas and hospital levels of China, a stronger network of diagnosis and treatment including all levels of hospitals across the country should be created to improve healthcare for rare diseases in the future.

\section{Conclusion}

In conclusion, this systematic review summarized the number, geographic and genetic characteristics of GSDs in Chinese biomedical publications. Analyzing number of the diseases revealed an imbalance in the distribution of areas and hospitals diagnosing rare diseases, which suggests that a multi-level network should be created to meet the specific challenge of healthcare for rare diseases in China.

\section{Competing interests}

The authors declare that they have no competing interests.

\section{Authors' contributions}

$\mathrm{JH}$ and $\mathrm{YC}$ put forward the idea and designed the key points. YC was responsible for the article writing and data analysis. $\mathrm{HZ}, \mathrm{ZL}$ and $\mathrm{CL}$ were responsible for the data collection. $\mathrm{HZ}$, JL and XZ participated in data analysis. All authors read and approved the final manuscript.

Received: 27 March 2012 Accepted: 20 August 2012

Published: 22 August 2012

\section{References}

1. Zelzer E, Olsen BR: The genetic basis for skeletal diseases. Nature 2003, 423:343-348.

2. Makitie O: Molecular defects causing skeletal dysplasias. Endocr Dev 2011, 21:78-84

3. Warman ML, Cormier-Daire V, Hall C, Krakow D, Lachman R, LeMerrer M, Mortier G, Mundlos S, Nishimura G, Rimoin DL, et al: Nosology and classification of genetic skeletal disorders: 2010 revision. Am J Med Genet A 2011, 155A:943-968.

4. Xia J, Wright J, Adams CE: Five large Chinese biomedical bibliographic databases: accessibility and coverage. Health Info Libr J 2008, 25:55-61.

5. Wang JB, Guo JJ, Yang L, Zhang YD, Sun ZQ, Zhang YJ: Rare diseases and legislation in China. Lancet 2010, 375:708-709.

6. Han JX, Cui YZ, Zhou XY: Rare diseases research in China: Opportunities, challenges, and solutions. Intractable Rare Dis Res 2012, 1:10-12.

7. Zhang YJ, Wang YO, Li L, Guo JJ, Wang JB: China's first rare-disease registry is under development. Lancet 2011, 378:769-770.

8. Aymé S, Gonthier C: Prevalence of rare diseases: Bibliographic data. Orphanet Report Series, Rare Diseases collection 2011, 1:1-29.

9. Zhou QH, Ling CQ, Bai YJ, Yin HX: Journal selection and indexing for Index Medicus and Chinese periodicals indexed in Index Medicus. Zhong Xi Yi Jie He Xue Bao 2005, 3:70-78.

10. Pace JM, Chitayat D, Atkinson M, Wilcox WR, Schwarze U, Byers PH: A single amino acid substitution (D1441Y) in the carboxyl-terminal propeptide of the proalpha1(I) chain of type I collagen results in a lethal variant of osteogenesis imperfecta with features of dense bone diseases. J Med Genet 2002, 39:23-29.
11. Liu Y: China's public health-care system: facing the challenges. Bull World Health Organ 2004, 82:532-538.

12. Ayme S, Schmidtke J: Networking for rare diseases: a necessity for Europe. Bundesgesundheitsblatt Gesundheitsforschung Gesundheitsschutz 2007, 50:1477-1483.

13. Black AP, Baker M: The impact of parent advocacy groups, the Internet, and social networking on rare diseases: the IDEA League and IDEA League United Kingdom example. Epilepsia 2011, 52(Suppl 2):102-104.

doi:10.1186/1750-1172-7-55

Cite this article as: Cui et al: A systematic review of genetic skeletal disorders reported in Chinese biomedical journals between 1978 and 2012. Orphanet Journal of Rare Diseases 2012 7:55.

\section{Submit your next manuscript to BioMed Central and take full advantage of:}

- Convenient online submission

- Thorough peer review

- No space constraints or color figure charges

- Immediate publication on acceptance

- Inclusion in PubMed, CAS, Scopus and Google Scholar

- Research which is freely available for redistribution 